\title{
Perfil das amostras do sistema nervoso central de bovinos com síndrome neurológica e diagnóstico da raiva bovina no serviço de defesa sanitária de Minas Gerais, 2003-2010 ${ }^{1}$
}

\author{
Taismara S. Oliveira ${ }^{2,4}$, Valquíria Bull ${ }^{3}$, Carolina A. Rezende ${ }^{3}$, Ronaldo Furtini ${ }^{4}$, \\ Érica A. Costa ${ }^{5}$, Tatiane A. Paixão ${ }^{6}$ e Renato L. Santos ${ }^{7^{*}}$
}

\begin{abstract}
Oliveira T.S., Bull V., Rezende C.A., Furtini R., Costa E.A., Paixão T.A. \& Santos R.L. 2012. [Profile of central nervous system samples from cattle with neurological syndrome and diagnosis of bovine rabies by the service of sanitary defense of Minas Gerais, 2003-2010.] Perfil das amostras do sistema nervoso central de bovinos com síndrome neurológica e diagnóstico da raiva bovina no serviço de defesa sanitária de Minas Gerais, 2003-2010. Pesquisa Veterinária Brasileira 32(4):333-339. Setor de Patologia Veterinária, Escola de Veterinária, Universidade Federal de Minas Gerais, Av. Antônio Carlos 6627, Cx. Postal 567, Pampulha, Belo Horizonte, MG 30161-970, Brazil. E-mail: rsantos@vet.ufmg.br

Diseases of the central nervous system (CNS) are often reported in cattle in Brazil. Although the State of Minas Gerais has the second largest cattle herd in the country, there is little information from this state concerning neurological diseases that affect cattle. The Laboratório de Saúde Animal of the Instituto Mineiro de Agropecuária (LSA/IMA) is in charge of the diagnosis of neurological diseases of livestock in the State, with emphasis on rabies and transmissible spongiform encephalopathies. A retrospective study was conducted on data from cattle with neurologic diseases evaluated by the LSA/IMA from January/2003 to June/2010, aiming to determine the profile of CNS samples sent for analysis, with emphasis on the diagnosis of bovine rabies. Issues related to the animal (sex, age, breed and type of death) as well as to the sample (method of conservation, person in charge the collection, and region of the CNS sampled) were evaluated. Data on frequency of rabies positive samples were analyzed by Fisher's exact test. During the period studied, 3,731 samples from cattle with neurological signs were analyzed, with a predominance of females and crossbred cattle. The method of preservation was the main problem encountered with only $25.89 \%$ of samples sent both refrigerated and fixed in $10 \%$ formaldehyde. There was a gradual decrease in submission of samples during the course of this study. All 3,703 samples were evaluated by direct fluorescent antibody test (DFA) and biological test (BT) for rabies, $41.58 \%$ being positivity for rabies, and 282 of those samples being subjected to histopathology examination. The frequency of positivity was influenced by breed, age, and type of death. Composition of the sample significantly influenced the results, with higher frequency of positivity in samples containing three or more CNS fragments by DFA, BT, or histopathology. The medulla, which is the fragment of choice for diagnosis of BSE, has often been mistakenly submitted under refrigeration, but not in $10 \%$ formalin. Cerebellum, thalamus, brain stem, and spinal cord had higher frequency of Negri bodies than
\end{abstract}

${ }^{1}$ Recebido em 26 de novembro de 2011.

Aceito para publicação em 16 de janeiro de 2012.

${ }^{2}$ Pós-graduanda de Mestrado em Ciência Animal, Escola de Veterinária da Universidade Federal de Minas Gerais (EV-UFMG), Av. Antônio Carlos 6627, Caixa Postal 567, Pampulha, Belo Horizonte, MG 30161970, Brasil.

${ }^{3}$ Bolsista de Iniciação Científica, EV-UFMG, Pampulha, Belo Horizonte, MG.

${ }^{4}$ Fiscal Agropecuário do Laboratório de Saúde Animal, Instituto Minei- ro de Agropecuária, Av. Contorno 1707A, Floresta, Belo Horizonte, MG 30110-005.

${ }^{5}$ Pós-doutoranda, EV-UFMG, Pampulha, Belo Horizonte, MG.

${ }^{6}$ Docente, Instituto de Ciências Biológicas da UFMG, Av. Antônio Carlos, 6627, Pampulha, Belo Horizonte, MG 31270-901.

${ }^{7}$ Docente, EV-UFMG, Avenida Antônio Carlos, 6627, Caixa Postal 567, Pampulha, Belo Horizonte, MG 30161-970. *Autor para correspondência: rsantos@vet.ufmg.br 
the cerebral cortex and trigeminal ganglia. The nonsuppurative inflammatory infiltrate was less frequent in the cerebral cortex than in other CNS fragments. In conclusion, CNS samples from cattle with neurological syndrome sent to the animal health protection service of Minas Gerais are heterogeneous, and the preservation method was the major problem hindering and adequate diagnosis. In addition, diagnosis of rabies was influenced by parameters of the animal as well as the CNS sample. Submission of properly preserved fragments from various segments of the CNS contributes to a more accurate diagnosis of rabies in cattle.

INDEX TERMS: Central nervous system, rabies, cattle, Minas Gerais.

RESUMO.- As enfermidades do sistema nervoso central (SNC) são frequentemente relatadas em bovinos no Brasil. Apesar de Minas Gerais ter o segundo maior rebanho bovino do país, há escassez de informações referentes às doenças neurológicas que acometem esses animais. 0 Laboratório de Saúde Animal do Instituto Mineiro de Agropecuária (LSA/IMA) é o responsável pelo diagnóstico das enfermidades neurológicas dos animais de produção no Estado, com ênfase para a raiva e as encefalopatias espongiformes transmissíveis. Foi realizado um estudo retrospectivo dos dados referentes às amostras de SNC de bovinos com síndrome neurológica avaliadas pelo LSA/IMA de janeiro/2003 a junho/2010, com o objetivo de determinar o perfil das amostras encaminhadas para análise no serviço de defesa sanitária animal, com ênfase no diagnóstico da raiva bovina. Foram consideradas características do animal (sexo, idade, raça e tipo de morte) e da amostra (método de conservação e responsável pela coleta), sendo nas positivas para raiva, avaliada sua composição, assim como as alterações histopatológicas encontradas. Os dados relacionados à frequência de positividade nas diferentes categorias foram submetidos à análise pelo Teste Exato de Fisher. Durante o período avaliado, foram analisadas 3.731 amostras de bovinos com doença neurológica, havendo predomínio de fêmeas e mestiços, o que reflete a composição do rebanho do Estado. 0 método de conservação foi o principal problema encontrado, sendo apenas $25,89 \%$ das amostras encaminhadas em gelo e formol a $10 \%$. Verificou-se uma diminuição gradativa no envio de material para análise. Quanto a raiva bovina diagnosticada no Estado, foram avaliadas 3.703 amostras pela imunofluorescência direta (IFD) e prova biológica (PB), com $41,58 \%$ de positividade, sendo dessas 282 submetidas a histopatologia. A frequência de positividade foi influenciada pela raça, idade e tipo de morte do animal. A composição da amostra alterou significativamente o resultado das análises, havendo maior frequência de positividade naquelas compostas por três ou mais fragmentos de SNC, tanto na IFD/PB, quanto na histopatologia. 0 bulbo, fragmento de eleição para o diagnóstico da EEB, tem sido erroneamente enviado refrigerado e não em formol a $10 \%$. Cerebelo, tálamo, tronco encefálico e medula apresentaram maior frequência de corpúsculos de $\mathrm{Ne}$ gri que cérebro e gânglio trigeminal. 0 infiltrado inflamatório não supurado foi menos frequente no cérebro, que nos demais fragmentos avaliados. Conclui-se que as amostras de bovinos com síndrome neurológica enviadas ao serviço de defesa sanitária animal de Minas Gerais apresentam características distintas, sendo o método de conservação o principal problema encontrado. Além disso, a raiva bovina diagnosticada na população estudada é influenciada pelas características do animal e da amostra, sendo indicado o envio de diferentes fragmentos do SNC para análise, conservados adequadamente, o que contribui para um diagnóstico mais preciso.

TERMOS DE INDEXAÇÃO: Sistema nervoso central, EETs, raiva, bovinos, Minas Gerais.

\section{INTRODUÇÃO}

As enfermidades do sistema nervoso central (SNC) são frequentemente relatadas em bovinos no Brasil, sendo causa importante de mortalidade em rebanhos e de grandes prejuízos econômicos (Riet-Correa et al. 1998, 2006, Salvador et al. 1998, Sanches et al. 2000, Garmatz et al. 2004, Karam et al. 2004, Lima et al. 2005, Galiza et al. 2010). Desde a descrição da encefalopatia espongiforme bovina no Reino Unido (Wells et al. 1987), relacionada posteriormente com uma variante da doença de Creutzfeldt-Jacob no homem (Trevitt \& Singh 2003), o diagnóstico definitivo das enfermidades que acometem o SNC de bovinos tornou-se ainda mais importante com intuito de cumprir as exigências sanitárias internacionais (Barros \& Marques 2003).

Apesar de Minas Gerais apresentar o segundo maior rebanho bovino do país, com um efetivo de 22.369 .639 cabeças (Produção 2008), há escassez de informações referentes às doenças neurológicas que acometem esses animais no Estado (Ferreira et al. 1986, Moro et al. 1994, Silva et al. 2001, Gomes et al. 2002, Ferreira 2006, Costa 2007, Dallora 2007, Menezes et al. 2008, Aquino Neto et al. 2009).

Segundo King \& Turner (1993), a raiva bovina resulta, em todo mundo, em perda anual de aproximadamente 50 milhões de dólares, sendo considerada uma das enfermidades mais importantes para a pecuária e para a saúde pública no Brasil (Schneider et al. 1996, Favoretto et al. 2002, Miranda et al. 2003, Lima et al. 2005). Estima-se que a raiva seja responsável anualmente pela morte de 30.000 a 40.000 bovinos no país, causando perdas anuais diretas estimadas em 15 milhões de dólares (Rodrigues da Silva et al. 2000, Heinemann et al. 2002). Além disso, há gastos indiretos com vacinações de animais e tratamentos pré e pós-exposição de humanos (Azevedo et al. 1981, Chamelet et al. 1982, Rodrigues da Silva et al. 2000).

Oficialmente, o diagnóstico da raiva é realizado através da técnica de imunofluorescência direta (IFD) e da prova biológica (PB), inoculação em camundongos ou células, a partir de amostras refrigeradas de SNC dos animais suspeitos (Brasil 2002b). Em Minas Gerais, o Laboratório de Saúde Animal do Instituto Mineiro de Agropecuária (LSA/ 
IMA) realiza o diagnóstico da raiva desde 1987 com ênfase em animais de produção. Durante esse período, estudos epidemiológicos foram realizados avaliando a distribuição espacial da doença no Estado ou sua ocorrência em determinados municípios (Silva et al. 2001, Ferreira 2006, Dallora 2007, Menezes et al. 2008). Porém, uma caracterização mais específica da doença nos bovinos, considerando categorias dos animais acometidos (raça, sexo ou idade) ainda não foi realizada. Além disso, não há estudos no Brasil avaliando a frequência de positividade de diferentes regiões do SNC de bovinos submetidas ao diagnóstico laboratorial de raiva (IFD e PB).

O Ministério da Agricultura, Pecuária e Abastecimento (MAPA) incorporou, em 2002, as Encefalopatias Espongiformes Transmissíveis (EETs) ao sistema de vigilância da raiva tornando obrigatória sua vigilância epidemiológica e o envio de parte do SNC conservado em formol a $10 \%$, juntamente com a porção refrigerada, permitindo a execução do diagnóstico diferencial (Brasil 2002b). 0 LSA/IMA também é responsável pela realização do diagnóstico das EETs no serviço de defesa sanitária de Minas Gerais. Nenhum estudo relacionado ao perfil das amostras de bovinos com síndrome neurológica encaminhadas para análise no LSA/ IMA foi realizado até o presente.

Diante do exposto, o objetivo desse trabalho é avaliar o perfil das amostras de SNC de bovinos com histórico de síndrome neurológica enviadas de janeiro de 2003 a junho de 2010 para análise no serviço de defesa sanitária animal de Minas Gerais, com ênfase no diagnóstico da raiva bovina.

\section{MATERIAL E MÉTODOS}

\section{Amostragem}

Amostras de SNC provenientes de bovinos que morreram após sintomatologia neurológica, foram coletadas em Minas Gerais por médicos veterinários autônomos e oficiais e encaminhadas ao LSA/IMA no período de janeiro de 2003 a junho de 2010. As amostras foram enviadas refrigeradas e/ou em formol a $10 \%$, acompanhadas de formulário contendo as informações referentes a cada uma, conforme preconizado por Barros \& Marques (2003).

As amostras foram submetidas às análises de imunofluorescência direta, prova biológica para raiva e histopatologia. A execução das técnicas laboratoriais foi dependente do tipo de conservação da mesma, ou seja, nem sempre a mesma amostra foi avaliada por todas as técnicas, tendo sido raiva 3.703 amostras processadas para IFD, 2.204 amostras submetidas a PB e 282 amostras processadas para histopatologia.

\section{Imunofluorescência direta e prova biológica para o diagnós- tico da raiva}

As amostras de SNC encaminhadas sob refrigeração foram submetidas aos exames de IFD segundo preconizado por Dean et al. (1996) e PB conforme descrito por Koprowski (1996). Também foi realizada histopatologia dos casos que continham amostras fixadas, conforme descrito abaixo. As amostras foram previamente fracionadas em segmentos específicos (córtex, hipocampo, cerebelo, tálamo, gânglio trigeminal, mesencéfalo, ponte, bulbo ou medula cervical), conforme enviados, para realização da IFD. As amostras foram classificadas em positivas e negativas para cada análise. Foi considerado diagnóstico negativo para raiva as amostras com resultados negativos na IFD e PB, e diagnóstico positivo para raiva, as amostras com resultado positivo em um dos testes.

\section{Histopatologia}

As amostras de SNC encaminhadas em formol a $10 \%$, que apresentaram resultado positivo para raiva (IFD e/ou PB), foram processadas pela técnica rotineira de inclusão em parafina, submetidas a microtomia seriada e coloração pela técnica da hematoxilina-eosina - HE (Luna 1968), para avaliação microscópica. As amostras foram previamente seccionadas em segmentos específicos (cérebro, cerebelo, tálamo, gânglio trigeminal, mesencéfalo, ponte, bulbo ou medula), conforme disponibilidade destes segmentos na composição das amostras. Os cortes histológicos foram classificados quanto à presença de infiltrado inflamatório não supurado e/ou de corpúsculo de Negri. Considerou-se diagnóstico conclusivo para raiva, quando o corpúsculo de Negri foi observado no fragmento.

\section{Levantamento e análise de dados}

Os dados obtidos nos formulários referentes aos parâmetros do animal, como sexo, idade, raça, tipo de morte (morte natural/ sacrifício) e município de origem; bem como aqueles referentes aos parâmetros da amostra, como método de conservação (gelo ou formol a $10 \%$ ) e técnico responsável pela coleta (veterinário autônomo ou do serviço de defesa sanitária oficial) foram catalogados e analisados.

Foram avaliadas a frequência de positividade da amostra na IFD e/ou PB para o diagnóstico de raiva bovina e sua distribuição de acordo com sexo, idade, raça, tipo de morte (morte natural/ sacrifício) e fragmentos do SNC analisados. A frequência de diagnósticos conclusivos à histopatologia (sensibilidade do teste) e a frequência de alterações histopatológicas sugestivas de raiva (infiltrado inflamatório não supurado e corpúsculo de Negri) também foram determinadas nos fragmentos analisados.

\section{Análise estatística}

Os dados referentes às frequências de positividade nas diferentes categorias avaliadas foram submetidos à análise estatística pelo Teste Exato de Fisher.

\section{RESULTADOS E DISCUSSÃO}

\section{Perfil das amostras encaminhadas para análise labora- torial}

Durante o período de janeiro de 2003 a junho de 2010 foram encaminhadas 3.731 amostras de bovinos com síndrome neurológica ao LSA/IMA para análise.

Avaliando as características relacionadas aos animais, houve predomínio de fêmeas $(2.499 / 3.731$ - 66,98\%) e de bovinos mestiços (1796/3.731 - 48,13\%). Animais acima de 24 meses, idade mínima necessária para inclusão no sistema oficial de vigilância epidemiológica das EETs (Brasil 2002a), corresponderam a 55,2\%, sendo 830 animais com idade entre quatro e sete anos (categoria de maior valor epidemiológico). Bovinos apresentando morte natural foram mais frequentes $(81,2 \%)$ do que sacrificados, o que é desejável para o sucesso no diagnóstico de algumas enfermidades que acometem o SNC de bovinos.

Quanto à origem, os bovinos eram provenientes de 563 municípios do Estado. Embora o número de amostras enviadas por município tenha sido variável, $19,9 \%$ das cidades tiveram apenas uma amostra enviada para análise. Além disso, considerando que Minas Gerais tem $853 \mathrm{mu}-$ nicípios, 33,9\% dos municípios não enviaram amostras de bovino com sinais clínicos neurológicos ao serviço de defesa sanitária animal de 2003 a 2010. Maiores estudos rela- 
cionados a esses índices são necessários, como a avaliação de possíveis fatores relacionados a escassez de amostras de determinados municípios.

Ao avaliar o método de conservação do SNC, apenas $25,89 \%$ do total de amostras foram encaminhadas em gelo e formol a $10 \%$, permitindo a realização de todas as análises previstas pelo sistema de vigilância da raiva e das EETs (Barros \& Marques 2003). A maioria das amostras enviadas, $73,35 \%$ (2.738/3.731), estava sob refrigeração. Considerando-se que o sistema de vigilância da raiva foi regulamentado no Brasil em 1976 (Brasil 1976) e que a determinação do envio de amostras encefálicas em formol a $10 \%$ só ocorreu em 2002 (Brasil 2002b), o predomínio de amostras refrigeradas pode ser parcialmente justificado pela prática rotineira adquirida ao longo dos anos pelos veterinários de campo de enviar apenas material sob refrigeração. Tais fatos requerem maiores estudos no intuito de definir estratégias para incrementar o envio de amostras em ambos os métodos de conservação.

Outro achado relevante foi a diminuição gradativa no número de amostras enviadas ao LSA/IMA para análise (Fig.1). Tal tendência não pode pode ser atribuída à diminuição gradativa das doenças neurológicas de bovinos no Estado em consequência do aumento da cobertura vacinal contra raiva, já. que Minas Gerais detém um rebanho bovino com mais de 22 milhões de cabeças (Produção 2008), e, mesmo uma redução drástica na incidência das enfermidades neurológicas, não resultaria numa diminuição tão acentuada no número de amostras de bovinos com óbito devido à síndrome neurológica. è necessário que estudos sejam realizados para identificar os fatores desencadeantes de tal tendência e que medidas sejam tomadas no intuito de estimular o envio de material para diagnóstico laboratorial.

Os veterinários do serviço de defesa oficial foram responsáveis por $56,1 \%$ do total de coletas realizadas. No primeiro ano analisado, $66,14 \%$ (461/697) das amostras foram enviadas por veterinários do serviço de defesa oficial. Porém, o envio de amostras por veterinários autônomos apresentou um aumento relativo até 2006, culminando com uma equivalência entre as duas categorias (Fig.2).

Durante o período estudado, 3.703 amostras foram avaliadas pelas técnicas de imunofluorescência direta e prova biológica, sendo $41,58 \%$ positivas para raiva $(1.540 / 3.703$ amostras). Dentre as positivas, 282 amostras foram avaliadas também pela histopatologia.

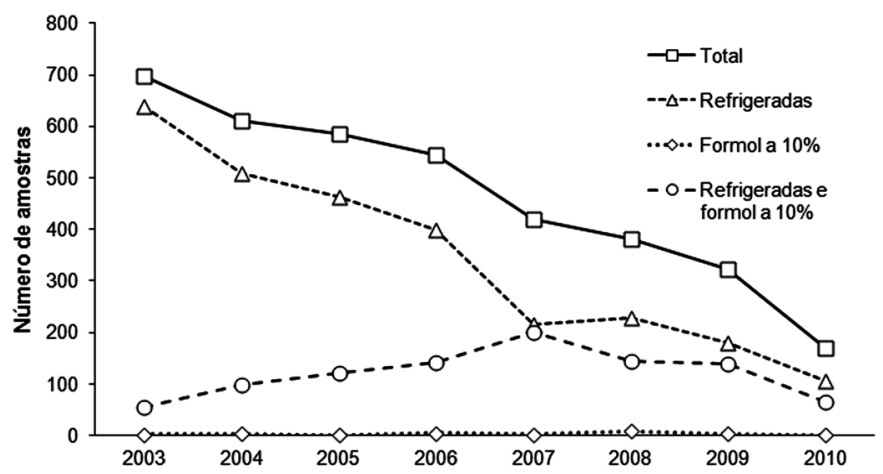

Fig.1. Distribuição temporal das 3.731 amostras de bovinos com síndrome neurológica enviadas ao LSA/IMA de 2003 a junho/2010 de acordo com o método de conservação.

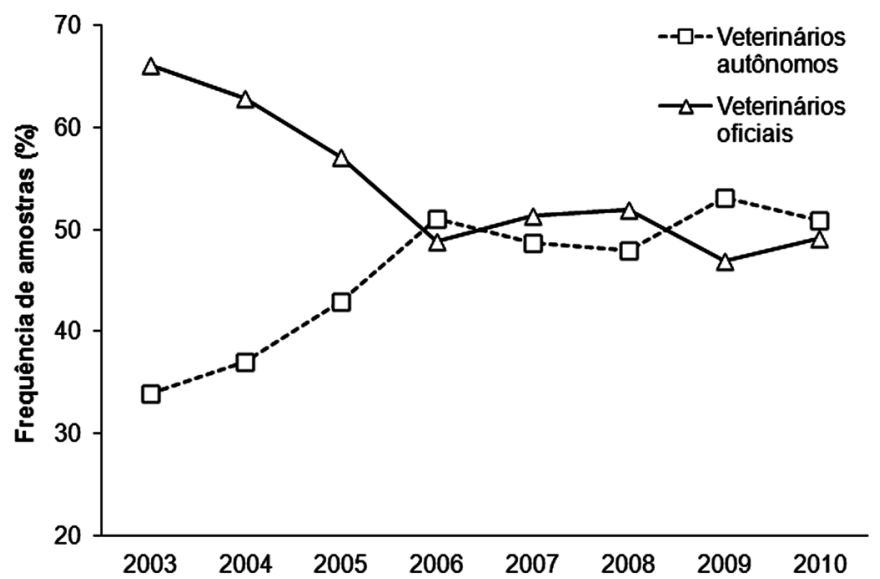

Fig.2. Frequência das amostras de bovinos com síndrome neurológica enviadas ao LSA/IMA de 2003 a junho/2010 segundo o responsável pela coleta.

Ao avaliar as características relacionadas aos animais, esse resultado não sofreu influência do sexo. Bos taurus taurus apresentaram maior frequência de positividade que Bos taurus indicus (33,8\% taurinos x 19,5\% zebuínos). Considerando-se as raças mais frequentes no estudo, a Nelore apresentou menor frequência de animais positivos que a Holandesa e os animais Girolando foram os de maior positividade, igualando-se aos mestiços (Quadro 1). Essa variação de positividade encontrada entre as raças pode estar relacionada aos diferentes tipos de criação e manejo, principalmente, considerando-se a maior positividade dos animais mestiços e Girolando sobre as raças puras.

Avaliando-se a faixa etária, animais até um mês e acima de 49 meses apresentaram menor frequência de positividade (Fig.3). Considerando-se que, a vacinação para raiva tem periodicidade anual (Brasil 2002b) e a natureza protéica complexa do vírus torna-o bom indutor de resposta imune (Kotait et al. 1998), a menor positividade de bovinos acima de 49 meses poderia ser justificada pela maior frequência de vacinação com o avançar da idade. Além disso, a capacidade de transferência de imunidade da vaca ao bezerro através do colostro (Brambell 1958) pode justificar o resultado apresentado pelos animais até um mês de idade. Os bezerros de 2 a 12 meses foram os de maior frequência de positividade, o que pode ser reflexo da não vacinação desses animais, devido ao nascimento entre as campanhas de vacinação anuais. Esses resultados devem ser mais bem

Quadro 1. Frequência de amostras positivas para raiva bovina na defesa sanitária de Minas Gerais de 2003 a junho/2010 de acordo com idade e raça

\begin{tabular}{lccccc}
\hline Raça & \multicolumn{4}{c}{ Idade $(\%)^{*}$} & \multirow{2}{*}{ Total $^{*}$} \\
\cline { 2 - 4 } & Até 1 mês & $2-12$ meses & meses & $>49$ meses & \\
\hline Holandês & $0(0)^{\mathrm{b}}$ & $51(39)^{\mathrm{a}}$ & $51(75)^{\mathrm{a}}$ & $16(20)^{\mathrm{b}}$ & \multirow{2}{*}{$38,86^{\mathrm{b}}(136)$} \\
Nelore & $33(1)^{\mathrm{a}}$ & $29(33)^{\mathrm{a}}$ & $15(44)^{\mathrm{b}}$ & $10(8)^{\mathrm{b}}$ & $17,29^{\mathrm{c}}(88)$ \\
Girolanda & $50(1)^{\mathrm{a}}$ & $61(66)^{\mathrm{a}}$ & $54(110)^{\mathrm{a}}$ & $32(47)^{\mathrm{b}}$ & $48,34^{\mathrm{a}}(233)$ \\
Mestiços & $27(5)^{\mathrm{b}}$ & $62(297)^{\mathrm{a}}$ & $50(428)^{\mathrm{a}}$ & $36(132)^{\mathrm{b}}$ & $49,5^{\mathrm{a}}(888)$ \\
\hline
\end{tabular}

$\overline{\mathrm{a}, \mathrm{b}, \mathrm{c}}$ Valores seguidos de letras diferentes na mesma linha apresentam diferença significativa $(\mathrm{p}<0,01)$.

* Valores entre parênteses representam o número absoluto de amostras positivas para raiva.

** Os valores totais de cada raça são maiores que os valores por idade somados, pois há amostras sem informações referentes à idade. 


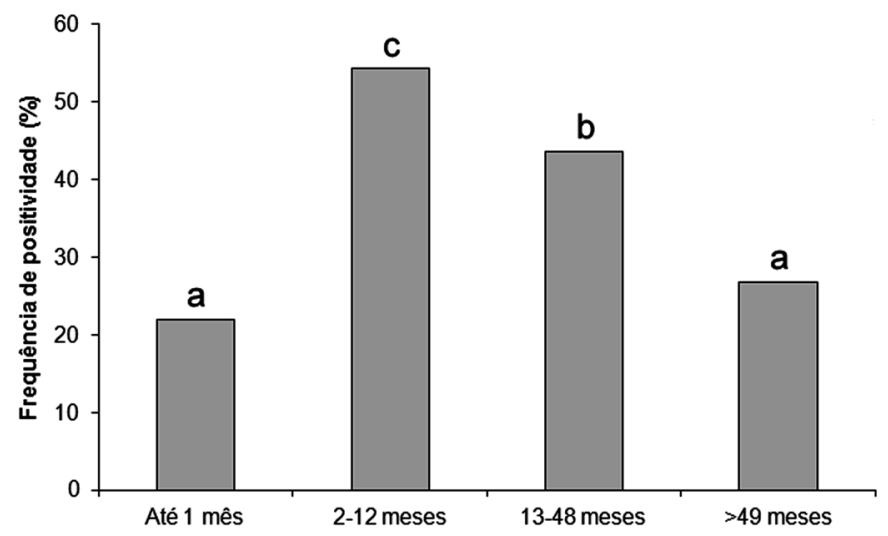

Fig.3. Frequência de diagnóstico da raiva bovina na defesa sanitária de Minas Gerais de 2003 a junho/2010 de acordo com idade. Colunas seguidas de letras diferentes apresentam diferença significativa $(\mathrm{p}<0,001)$.

estudados, no intuito de definir os fatores desencadeantes de tais achados. Outro achado relevante foi que essa distribuição da faixa etária alterou-se quando avaliada em cada raça citada anteriormente (Quadro 1), o que sugere um efeito conjunto entre raça e idade sobre a frequência de positividade para raiva na população estudada.

0 tipo de morte influenciou o resultado da imunofluorescência direta, apresentando os animais sacrificados menor positividade $(34,08 \%-137 / 402)$ que os bovinos com morte natural (41,66\% - 1261/3027).

A avaliação da composição da amostra revelou resultados relevantes (Quadro 2). Segundo recomendação do MAPA (Barros \& Marques 2003), o tronco encefálico, composto por mesencéfalo, ponte e bulbo, deve ser encaminhado íntegro e conservado em formol a 10\%, para análise histopatológica. Porém, verifica-se que a porcentagem de amostras formolizadas que continham tais fragmentos foi variável, indicando a não integridade do tronco encefálico em parte das amostras avaliadas. 0 bulbo, fragmento de eleição para o diagnóstico da Encefalopatia Espongiforme Bovina - EEB (Barros \& Marques 2003), foi enviado em $60,84 \%$ das amostras refrigeradas para diagnóstico da raiva e não estava presente em $35,82 \%$ das conservadas em formol. Tal achado requer especial atenção, exigindo maior

Quadro 2. Frequência de regiões do sistema nervoso central na composição das amostras de bovinos enviadas ao Serviço de Defesa Sanitária de Minas Gerais, de janeiro de 2003 a junho de 2010, conservadas sob refrigeração e em formol a $10 \%$

\begin{tabular}{lcc}
\hline & \multicolumn{2}{c}{ Método de conservação (\%) } \\
\cline { 2 - 3 } Regiões do SNC & $\begin{array}{c}\text { Amostras } \\
\text { refrigeradas }\end{array}$ & $\begin{array}{c}\text { Amostras em } \\
\text { formol a 10\% }\end{array}$ \\
\hline Cérebro & $98,23(2002)$ & $76,59(216)$ \\
Hipocampo & $72,27(1473)$ & $0,71(2)$ \\
Cerebelo & $67,17(1369)$ & $78,37(221)$ \\
Gânglio trigeminal & $0,0004(1)$ & $17,73(50)$ \\
Tálamo & $0,44(9)$ & $28,37(80)$ \\
Mesencéfalo & $0(0)$ & $50,71(143)$ \\
Ponte & $0(0)$ & $63,83(180)$ \\
Bulbo & $60,84(1240)$ & $64,18(181)$ \\
Medula & $0,05(104)$ & $10,99(31)$ \\
* Valores entre parênteses representam o número absoluto de amostras \\
\multicolumn{1}{c}{ contendo cada fragmento especificado. }
\end{tabular}

divulgação aos veterinários para a necessidade da remessa do bulbo em formol a 10\%. A execução das técnicas de histopatologia e imunohistoquímica, exames de eleição para o diagnóstico da EEB são feitas somente em fragmentos enviados formolizados (Barros \& Marques 2003). Além disso, a presença do cérebro ou do cérebro e cerebelo na grande maioria das amostras refrigeradas ou formolizadas respectivamente, reflete uma coleta ainda direcionada para fragmentos destas regiões sem priorizar coleta sistemática de diversas regiões do encéfalo, fundamental para diagnóstico definitivo das diversas afecções do SNC de bovinos.

$\mathrm{O}$ número de fragmentos de diferentes regiões do SNC enviados em cada amostra influenciou significativamente o diagnóstico da raiva. Não houve diferença significativa na frequência de positividade entre os diferentes fragmentos avaliados pela IFD e PB, contudo, amostras compostas somente por cérebro apresentaram menor frequência de positividade do que as amostras contendo cérebro associado a dois ou mais fragmentos de outras regiões do SNC nestes testes (Figura 4). Segundo preconizado por Barros \& Marques (2003), a calota craniana deve ser aberta em sua totalidade, seccionando os ossos frontal, temporal e occipital, o que permite a exposição de todo o encéfalo para coleta adequada dos fragmentos. Tal procedimento exige tempo, esforço e equipamentos específicos (serra comum, cutelo e tesoura). Durante a coleta a campo, os veterinários nem sempre tem a disposição equipamentos para tal feito, executando a abertura somente do osso frontal (mais delgado). Porém, esta abertura permite somente a coleta do cérebro, prejudicando a composição da amostra e podendo comprometer o diagnóstico final.

Na histopatologia (Quadro 3), cerebelo, tálamo, tronco encefálico e medula apresentaram maior frequência de corpúsculos de Negri (Fig.5a) que cérebro e gânglio trigeminal. Esse achado corrobora parcialmente trabalhos anteriores, que descreveram maior frequência de corpúsculos de Negri no cerebelo (Langohr et al. 2003, Pedroso et al.

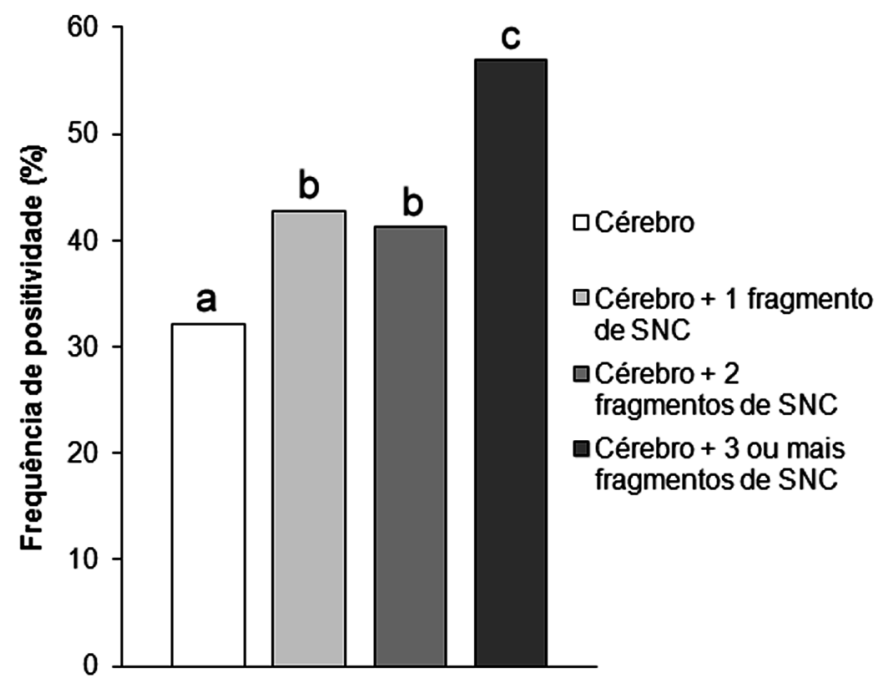

Fig.4. Frequência de positividade para raiva bovina pela imunofluorescência direta e prova biológica segundo a composição da amostra refrigerada. Colunas seguidas de letras diferentes apresentam diferença significativa $(\mathrm{p}<0,05)$. 
Quadro 3. Frequência das alterações histopatológicas (\%) sugestivas de raiva nas diferentes regiões do SNC

\begin{tabular}{lccc}
\hline \multirow{2}{*}{ Regiões do SNC } & \multicolumn{3}{c}{ Alterações histopatológicas } \\
\cline { 2 - 4 } & $\begin{array}{c}\text { Sem alterações } \\
\text { significativas }\end{array}$ & $\begin{array}{c}\text { Inflamação } \\
\text { não supurada }\end{array}$ & $\begin{array}{c}\text { Corpúsculos } \\
\text { de Negri }\end{array}$ \\
\hline Cérebro & $36,1^{\mathrm{a}}$ & $54,2^{\mathrm{c}}$ & $36,6^{\mathrm{b}}$ \\
Cerebelo & $21,2^{\mathrm{b}}$ & $67,0^{\mathrm{b}}$ & $53,9^{\mathrm{a}}$ \\
Gânglio trigeminal & $16,0^{\mathrm{b}}$ & $84,0^{\mathrm{ab}}$ & $18,0^{\mathrm{c}}$ \\
Tálamo & $12,5^{\mathrm{bc}}$ & $79,0^{\mathrm{b}}$ & $46,2^{\mathrm{a}}$ \\
Mesencéfalo & $10,5^{\mathrm{bc}}$ & $86,7^{\mathrm{a}}$ & $45,5^{\underline{\mathrm{a}}}$ \\
Ponte & $6,1^{\mathrm{c}}$ & $88,8^{\underline{\mathrm{a}}}$ & $50,0^{\underline{a}}$ \\
Bulbo & $7,2^{\mathrm{c}}$ & $87,8^{\mathrm{a}}$ & $47,0^{\mathrm{a}}$ \\
Medula & $9,6^{\mathrm{bc}}$ & $67,7^{\mathrm{b}}$ & $45,2^{\mathrm{a}}$
\end{tabular}

* Valores seguidos de letras diferentes nas colunas apresentam diferença significativa $(\mathrm{p}<0,05)$.

2009) e gânglio trigeminal (Rech et al. 2006, Pedroso et al. 2009). Esta diferença nos resultados pode ser justificada pela diferença no número de animais avaliados muito maior neste trabalho. 0 infiltrado inflamatório não supurado (Fig.5b), comum em infecções virais no SNC (Langohr et al. 2003), foi menos frequente no cérebro, que nos demais fragmentos avaliados (Quadro 3).

Semelhante ao observado nos testes IFD e PB, o número de amostras com diagnóstico conclusivo para raiva foi menor quando o cérebro foi analisado individualmente no exame histopatológico (Quadro 4). Além disso, a sensibilidade da histopatologia em amostras contendo apenas cé-

Quadro 4. Frequência de diagnósticos conclusivos para raiva bovina pela histopatologia de acordo com a composição da amostra em formol a $\mathbf{1 0 \%}$

\begin{tabular}{ll}
\hline \multicolumn{1}{c}{ Composição da amostra } & $\begin{array}{c}\text { Histopatologia, } \\
\text { sensibilidade (\%) }\end{array}$ \\
\hline Cérebro & $27,27(3)^{\mathrm{c}}$ \\
Cérebro + 01 fragmento aleatório de SNC & $57,69(15)^{\mathrm{bc}}$ \\
Cérebro + 02 fragmentos aleatórios de SNC & $71,88(23)^{\mathrm{ab}}$ \\
Cérebro + 03 fragmentos aleatórios de SNC & $67,35(33)^{\mathrm{ab}}$ \\
Cérebro + 04 ou mais fragmentos aleatórios de SNC & $80,21(77)^{\mathrm{a}}$ \\
* Valores seguidos de letras diferentes apresentam diferença significativa \\
(p<0,01) \\
** Valores entre parênteses representam o número absoluto de amostras \\
com diagnóstico conclusivo para raiva (presença do corpúsculo de Ne- \\
gri) segundo a composição da amostra.
\end{tabular}

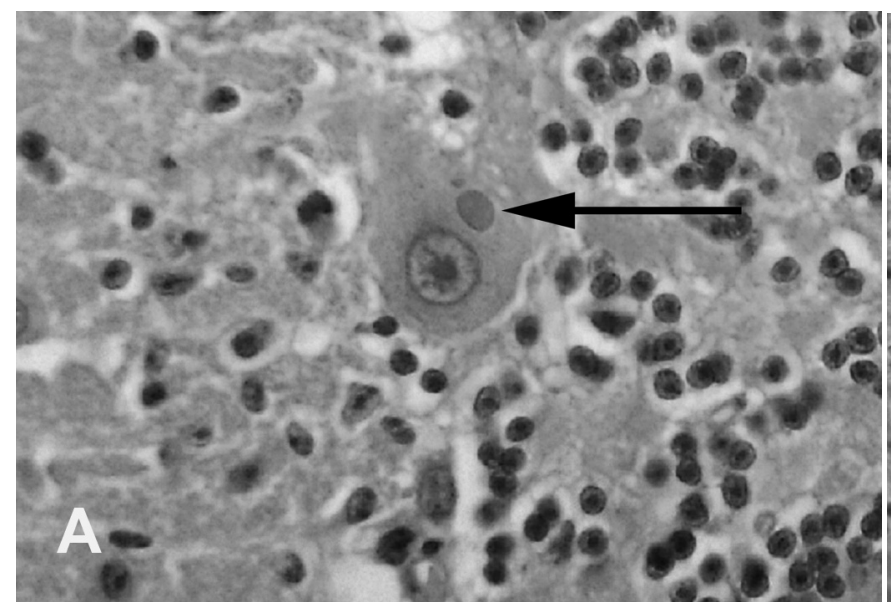

rebro $(27,2 \%$ - 3 diagnósticos conclusivos em 11 amostras avaliadas) foi extremamente menor do que nas amostras compostas por cérebro associado a quatro ou mais fragmentos de diferentes regiões do SNC $(80,2 \%$ - 77 diagnósticos conclusivos em 96 amostras avaliadas). Estes dados reforçam a importância da coleta de várias regiões do SNC de bovinos tanto em gelo quanto em formalina a $10 \%$ para obter sucesso no diagnóstico laboratorial.

\section{CONCLUSÕES}

O perfil das amostras de SNC de bovinos com síndrome neurológica, enviadas ao serviço de defesa sanitária animal de Minas Gerais de 2003 a junho de 2010, atende parcialmente os requisitos de vigilância da raiva e EETs. 0 método de conservação foi o principal problema encontrado, havendo predomínio de amostras refrigeradas e escassez de amostras formolizadas, inviabilizando a realização da histopatologia em boa parte delas.

Além disso, verificou-se um decréscimo gradativo no número total de amostras de bovinos com síndrome neurológica encaminhadas para análise durante o período estudado, fato que requer especial atenção pelos órgãos de defesa sanitária, no intuito de incrementar tal envio.

0 diagnóstico da raiva bovina nas amostras analisadas pelo serviço de defesa sanitária animal é influenciado pelas características do animal, com ênfase na raça, idade e tipo de morte.

A composição da amostra alterou significativamente a frequência de positividade para raiva, indicando-se o envio de no mínimo três fragmentos de diferentes regiões do SNC refrigerados e em formol a 10\%, para execução da imunofluorescência direta, prova biológica e histopatologia.

O bulbo - fragmento de eleição para o diagnóstico da EEB - tem sido erroneamente enviado refrigerado e não em formol a $10 \%$, inviabilizando a execução da histopatologia e imunohistoquímica (técnicas oficiais para o diagnóstico das EETs) em parte das amostras.

Esse estudo é inédito no serviço de defesa sanitária animal de Minas Gerais, trazendo resultados relevantes e altamente aplicáveis à rotina dos veterinários autônomos e oficiais durante a coleta de amostras encefálicas de bovinos.

Fig.5. Alterações histopatológicas sugestivas de raiva, no SNC de bovino. (A) Corpúsculo de Negri (seta) em célula de Purkinje do cerebelo. HE, obj.40x. (B) Infiltrado inflamatório não supurado, perivascular, intenso (manguito perivascular) em fragmento de ponte. HE, obj.40x. 
Agradecimentos.- Este trabalho teve o apoio do Ministério da Agricultura, Pecuária e Abastecimento (MAPA), do CNPq e da FAPEMIG. CAR, EAC e RLS são bolsistas do CNPq. VB é bolsista da FAPEMIG.

\section{REFERÊNCIAS}

Aquino Neto H.M., Carvalho A.U., Facury Filho E.J., Ferreira P.M., Barbosa-Stan E.F., Lobato Z.I.P., Alvarenga M.R., Serranno A.L., Martins R.A. \& Afonso D.A.F. 2009. Meningoencefalite por Herpesvirus bovino 5 em Minas Gerais: relato de caso clínico. Arq. Bras. Med. Vet. Zootec. 61(1):1-5.

Azevedo M.P., Barbante P.J., Kerbrie S.V. \& Cortás M.C. 1981. Tratamento preventivo da raiva humana. Revta Saúde Públ. 15(5):551-554.

Barros C.S.L. \& Marques G.H.F. 2003. Procedimentos para o diagnóstico das doenças do sistema nervoso central de bovinos. MAPA/SDA/DDA, Brasília. 50p.

Brambell J.W.R. 1958. The passive immunity of young mammal. Biol. Rev. 33:488-531.

Brasil 1976. Instrução Normativa № 126, de 18 de março de 1976, aprova as Normas anexas à presente Portaria, assinada pelo Diretor-Geral do Departamento Nacional de Produção Animal, a serem observadas na Profilaxia da Raiva. Diário Oficial da União de 6 de abril de 1976, Seção 1, p.4434.

Brasil 2002a. Instrução Normativa № 18, de 15 de fevereiro de 2002, aprova as Normas a serem adotadas, visando incrementar a vigilância epidemiológica para detecção de Encefalopatias Espongiformes Transmissíveis (EET) em ruminantes. Diário Oficial da União de 18 de fevereiro de 2002, Seção 1, p.1.

Brasil 2002b. Instrução Normativa ${ }^{\circ}$ 05, de 01 de março de 2002, a prova as Normas Técnicas para o controle da raiva dos herbívoros domésticos. Diário Oficial da União de 4 de março de 2002, Seção 1, p.3.

Chamelet E.L.B., Azevedo M.P., Favoretto S.R., Kerbie S.V. \& Souza L.T.M. 1982. Esquema reduzido de vacinação anti-rábica humana pré-exposição e avaliação de doses anuais de reforço. Revta Saúde Públ. 16(3):144-148.

Costa E.A. 2007. Diagnóstico das encefalites herpéticas em bovinos. Tese de Doutorado em Microbiologia Veterinária, Escola de Veterinária, Universidade Federal de Minas Gerais, Belo Horizonte, MG. 58p.

Dallora A.M.B. 2007. Estudo da ocorrência de casos confirmados de raiva bovina no município de Guaxupé, Minas Gerais. Dissertação de Mestrado em Saúde na Comunidade, Faculdade de Medicina de Ribeirão Preto, Ribeirão Preto, SP. 91p.

Favoretto S.R., Carrieri M.L., Cunha E.M.S., Aguiar E.A.C, Silva L.H.Q., Sodré M.M., Souza M.C.A.M. \& Kotait I. 2002. Tipificação antigênica de amostras brasileiras de vírus rábico isoladas de animais e humanos, no período de 1989 a 2000. Revta Inst. Med. Trop., S. Paulo, 44(2):91-95.

Ferreira R.S. 2006. Levantamento epidemiológico da Raiva no estado de Minas Gerais, no período de 2002 a 2006. Dissertação de Mestrado em Ciência Animal, Unifenas, Alfenas, MG. 82p.

Ferreira F.A., Coelho H.E. \& Bastos J.E.D. 1986. Polioencefalomalácia em bovinos no estado de Minas Gerais. Arq. Bras. Med. Vet. Zootec. 38:693-700.

Galiza G.J.N., Silva, M.L.C.R., Dantas, A.F.M., Simões, S.V.D. \& Riet-Correa F. 2010. Doenças do sistema nervoso de bovinos no semiárido nordestino. Pesq. Vet. Bras. 30(3):267-276.

Garmatz S.L., Irigoyen L.F., Rech R.R., Brown C. C., Zhang J. \& Barros C.S.L. 2004. Febre catarral maligna em bovinos no Rio Grande do Sul: transmissão experimental para bovinos e caracterização do agente etiológico. Pesq. Vet. Bras. 24(2):93-103.

Gomes L.I., Rocha M.A., Costa E.A., Lobato Z.I.P., Mendes L.C.N., Borges A.S., Leite R.C. \& Barbosa-Stancioli E.F. 2002. Detecção de herpesvírus bovino 5 (BoHV-5) em bovinos do Sudeste Brasileiro. Arq. Bras. Med. Vet. Zootec. 54(2):217-220.

Heinemann F.M., Fernandes-Matioli F.M.C., Cortez A., Soares R.M., Sakamoto S.M., Bernardi F., Ito F.H., Madeira A.M.B.N. \& Richtzenhain L.J. 2002. Genealogical analysis of rabies virus strain from Brazil based on $\mathrm{N}$ gene alleles. Epidemiol. Infect. 128:503-511.
Karam F.S.C., Soares M.P., Haraguchi M., Riet-Correa F., Méndez M.C. \& Jarenkow J.A. 2004. Aspectos epidemiológicos da seneciose na região sul do Rio Grande do Sul. Pesq. Vet. Bras. 24(4):191-198.

King A.A. \& Turner G.S. 1993. Rabies: A review. J. Comp. Pathol. 108:139.

Kotait I., Gonçalves C.A., Peres N.F., Souza M.C.A.M. \& Targueta M.C. 1998. Controle da raiva dos herbívoros. Manual Técnico do Instituto Pasteur, São Paulo, 1:15.

Langohr I.M., Irigoyen L.F., Lemos R.A.A. \& Barros C.S.L. 2003. Aspectos epidemiológicos, clínicos e distribuição das lesões histológicas no encéfalo de bovinos com raiva. Ciência Rural 33(1):125-131.

Lima E.F., Riet-Correa F., Castro R.S., Gomes A.A.B. \& Lima F.S. 2005. Sinais clínicos, distribuição das lesões no sistema nervoso e epidemiologia da raiva em herbívoros na região Nordeste do Brasil. Pesq. Vet. Bras. 25(4):250-264.

Menezes F.L., Silva J.A., Moreira E.C., Meneses J.N.C., Magalhães D.F., Barbosa A.D. \& Oliveira C.S.F. 2008. Distribuição espaço-temporal da raiva bovina em Minas Gerais, 1998 a 2006. Arq. Bras. Med. Vet. Zootec. 60(3):566-573.

Miranda C.F.J., Silva J.A. \& Moreira E.C. 2003. Raiva humana transmitida por cães: áreas de risco em Minas Gerais, Brasil, 1991-1999. Cad. Saúde Públ. 19(1):91-99.

Moro L., Nogueira R.H.G., Carvalho A.U. \& Marques D.C. 1994. Relato de três casos de polioencefalomalacia em bovinos. Arq. Bras. Med. Vet. Zootec. 46(4):409-416.

Pedroso P.M.O., Colodel E.M., Pescador C.A., Arruda L.P. \& Driemeier D. 2009. Aspectos clínicos e patológicos em bovinos afetados por raiva com especial referência ao mapeamento do antígeno rábico por imuno-histoquímica. Pesq. Vet. Bras. 29(11):899-904.

Produção da Pecuária Municipal 2008. Instituto Brasileiro de Geografia e Estatística, Rio de Janeiro, RJ, 36:1-55.

Rech R., Rissi D.R., Silva M.C., Inkelmann M.A. \& Barros C.S.L. 2006. Histomorfologia do gânglio de Gasser, da rete mirabile carotídea e da hipófise de bovinos: estudo de 199 casos. Pesq. Vet. Bras. 26(2):105-111.

Riet-Correa F., Schild A.L. \& Fernandes C.G. 1998. Enfermidades do sistema nervoso dos ruminantes no sul do Rio Grande do Sul. Ciência Rural 28(2):341-348.

Riet-Correa G., Duarte M.D., Barbosa J.D., Oliveira C.M.C., Cerqueira V.D., Brito M.F. \& Riet-Correa F. 2006. Meningoencefalite e polioencefalomalacia causadas por Herpesvírus bovino-5 no estado do Pará. Pesq. Vet. Bras. 26(1):44-46.

Rodrigues da Silva A.D.C., Caporale G.M.M., Gonçalves C.A., Targueta M.C., Comin F., Zanetti C.R. \& Kotait I. 2000. Antibody response in cattle after vaccination with inactivated and attenuated rabies vaccines. Revta Inst. Med. Trop. 42:95-98.

Salvador S.C., Lemos R.A.A., Riet-Correa F., Roehe P.M. \& Osório A.L.A.R. 1998. Meningoencefalite em bovinos causada por herpesvírus bovino-5 no Mato Grosso do Sul e São Paulo. Pesq. Vet. Bras. 18(2):76-83.

Sanches A.W.D., Langohr I.M., Stigger A.L. \& Barros C.S.L. 2000. Doenças do sistema nervoso central em bovinos no Sul do Brasil. Pesq. Vet. Bras. 20(3):113-118.

Schneider M.C., Almeida G.A., Souza L.M., Morares N.B. \& Diaz R.C. 1996. Controle da raiva no Brasil de 1980 a 1990. Revta Saúde Públ. 30(2):196-203.

Silva J.A., Moreira E.C., Haddad J.P.A., Modena C.M. \& Tubaldini M.A.S. 2001. Distribuição temporal e espacial da raiva bovina em Minas Gerais, 1976 a 1997. Arq. Bras. Med. Vet. Zootec. 53(3):263-272.

Trevitt C.R. \& Singh P.N. 2003. Variant Creutzfeldt-Jacob disease: Pathology, epidemiology and public health implications. Am. J. Clin. Nutr. 78:651-656.

Wells G.A.H., Scott A.C., Johnson C.T., Gunning R.F., Hancock R.D., Jeffrey M., Dawson M. \& Bradley R. 1987. A novel progressive spongiform encephalopathy in cattle. Vet. Rec. 121:419-420. 